Research Article

\title{
Degree-Based Topological Indices of Boron $B_{12}$
}

\author{
Nouman Saeed $\mathbb{D},{ }^{1}$ Kai Long $\mathbb{D},{ }^{1}$ Zeeshan Saleem Mufti $\mathbb{D}^{2},{ }^{2}$ Hafsa Sajid, \\ and Abdul Rehman $\mathbb{D D}^{2}$
}

${ }^{1}$ State Key Laboratory for Alternate Electrical Power System with Renewable Energy Sources, North China Electric Power University, Beijing 102206, China

${ }^{2}$ The University of Lahore, Lahore Campus, Lahore, Pakistan

Correspondence should be addressed to Kai Long; longkai1978@163.com

Received 11 February 2021; Revised 1 March 2021; Accepted 22 March 2021; Published 29 March 2021

Academic Editor: Kashif Ali

Copyright $\odot 2021$ Nouman Saeed et al. This is an open access article distributed under the Creative Commons Attribution License, which permits unrestricted use, distribution, and reproduction in any medium, provided the original work is properly cited.

Topological index sometimes called molecular descriptor is a numerical value which associates a chemical composition for correlating chemical structure with numerous physical properties, chemical reactivity, or biological activity. In this paper, we study some topological indices of boron and try to correlate the physicochemical properties such as freezing points, boiling points, melting points, infrared spectrum, electronic parameters, viscosity, and density of chemical graphs. We discuss these topological indices, and some of them are mentioned here such as Randic index, the first general Zagreb index, the general sum connectivity index, hyper-Zagreb index (HM), the atom-bond connectivity index (ABC), the geometric-arithmetic index (GA), the harmonic index $(H)$, and the forgotten index $(F)$.

\section{Introduction and Applications}

A chemical graph theory is an outlet of mathematical chemistry which applied graph theory to the molecular structure of chemical compounds. Topological index is a part of chemical graph theory which correlates the physicochemical properties such as freezing point, boiling point, melting point, infrared spectrum, electronic parameters, viscosity, and density of the underlying chemical graphs. The reader can find bulk of papers on topological indices which have been in print so far [1-5].

A graph can be recognized by a polynomial, a matrix, a sequence of numbers, or a numeric number which represents the whole graph, and these representations are designed to be uniquely defined for that graph. Topological indices are major tools for analyzing many physicochemical properties of molecules without performing any testing. Some most significant types of topological indices of graphs are distance-based topological indices, degree-based topological indices, and spectrum-based topological indices. One of the most investigated categories of topological indices used in mathematical chemistry is called degree-based topological indices, which are defined in terms of the degrees of the vertices of a graph.

Topological index is a graph invariant which characterizes the topology of the molecular structure and converts the molecular graph into a real number that predicts some physicochemical properties such as freezing point, boiling point, and melting point. Nowadays, a biological testing of chemical compounds is too much expensive. It requires a very large laboratory and advanced equipment to test these compounds. This process is costly and time-consuming. Due to this factor, pharmaceutical companies are very eager to find such new ideas or methods by which cost could be reduced. One can reduce the cost in which no need of laboratories and no need of equipment, but just need to study the certain chemical structure using topological indices

Topological indices are of different types such as degreebased topological indices, distance-based topological indices, and spectrum-based topological indices. The notion of topological index was discovered in 1947 when Harold Wiener was working on the boiling point of paraffin. $\mathrm{He}$ named this index as path number. Later on, the path number 
was renamed as the Wiener index, and then the theory of topological index started. The Wiener index is the first and the most studied topological index. Wiener index is defined as the sum of distances among all the sets of vertices in $G$ [6]. Among all the types of topological indices, degree-based topological indices play an extensive role in chemical graph theory [7]. That motivated us to study the chemical structure of boron $\mathrm{B}_{12}$ under this phenomenon.

$\mathrm{B}_{12}$ is a two-dimensional icosahedral network. A recent study of high-pressure solid boron affirmed that the icosahedral $\mathrm{B}_{12}$-containing structures are quite universal [8]. On the other hand, previous experimental and theoretical studies of freestanding boron clusters have shown that the $\mathrm{B}_{12}$ structure is unstable in the gas phase $[9,10]$. The $(n, m)$ unit of boron $\mathrm{B}_{12}$ structure is given in the figure, where " $n$ " represents the number of rows and " $m$ " represents the number of columns of the boron $\mathrm{B}_{12}$ structure.

Let $G$ be a simple graph with order $p$ and size $q$. Let $d_{v}$ represent the degree of the vertex $v$ and is defined as the number of edges incident on $v$. Let $S_{v}$ represent the sum of the degree of all the neighbors of $v$ [11]. Graph theory is closely connected to the computer science, applied mathematics, optimization theory, web designing, operation research, biology, and chemistry.

The general Randic connectivity index of $G$ is defined as

$$
R_{\alpha}(G)=\sum_{r s \in E(G)}\left(d_{r} d_{s}\right)^{\alpha},
$$

where $\alpha$ represents a real number. If $\alpha$ is $-1 / 2$, then $R_{-1 / 2}(G)$ is said to be the Randic connectivity index of $G$. Gutman and Trinajstic presented the first general Zagreb index in 1972 [12]:

$$
M_{\alpha}(G)=\sum_{r \in V(G)}\left(d_{r}\right)^{\alpha} .
$$

In 2010, the general sum connectivity index $\chi_{\alpha}(G)$ has been invented:

$$
\chi_{\alpha}(G)=\sum_{r s \in E(G)}\left(d_{r}+d_{s}\right)^{\alpha} .
$$

The $A B C$ index was presented by Estrada and Torres et al. in [13]. The $\mathrm{ABC}$ index of graph $G$ is expressed as

$$
\operatorname{ABC}(G)=\sum_{r s \in E(G)} \sqrt{\frac{d_{r}+d_{s}-2}{d_{r} d_{s}}} .
$$

Das announced the geometric-arithmetic (GA) index in $[14,15]$. The geometric-arithmetic index denoted by GA for graph $G$ is presented by

$$
\mathrm{GA}(G)=\sum_{r s \in E(G)} \frac{2 \sqrt{d_{r} d_{s}}}{d_{r}+d_{s}} .
$$

In 2013, the hyper-Zagreb index has been introduced by Shirdel et al. as

$$
\operatorname{HM}(G)=\sum_{r s \in E(G)}\left(d_{r}+d_{s}\right)^{2} .
$$

In 2012, Zhang introduced the harmonic index. It is defined as follows [16]:

$$
H(G)=\sum_{r s \in E(G)} \frac{2}{\left(d_{r}+d_{s}\right)} .
$$

After working on the Zagreb index, in 2015, Furtula and Gutman introduce the forgotten index $F(G)$ :

$$
F(G)=\sum_{r s \in E(G)}\left(d_{r}^{2}+d_{s}^{2}\right) .
$$

Ghorbani and Azimi proposed two new types of Zagreb indices of a graph $G$ in 2012. $\mathrm{PM}_{1}$ is the first multiple Zagreb index, and $\mathrm{PM}_{2}$ is the second multiple Zagreb index [6]:

$$
\begin{aligned}
& \operatorname{PM}_{1}(G)=\prod_{r s \in E(G)}\left(d_{r}+d_{s}\right), \\
& \operatorname{PM}_{2}(G)=\prod_{r s \in E(G)}\left(d_{r} \times d_{s}\right),
\end{aligned}
$$

$M_{1}(G, p)$ and $M_{2}(G, p)$, the first Zagreb polynomial and the second Zagreb polynomial [12, 17], respectively, are defined as

$$
\begin{aligned}
& M_{1}(G, p)=\sum_{r s \in E(G)} p^{\left(d_{r}+d_{s}\right)}, \\
& M_{2}(G, p)=\sum_{r s \in E(G)} p^{\left(d_{r} \times d_{s}\right)} .
\end{aligned}
$$

Recently, Furtula et al. proposed the second Zagreb index:

$$
\mathrm{RM}_{2}(G)=\sum_{r s \in E(G)}\left(d_{r}-1\right)\left(d_{s}-1\right) .
$$

After the success of the $A B C$ index, Furtula et al. put forward its modified version in 2010 that they somewhat inadequately named "augmented Zagreb index." It is defined as follows [18]:

$$
\operatorname{AZI}(G)=\sum_{r s \in E(G)}\left(\frac{d_{r} d_{s}}{d_{r}+d_{s}-2}\right)^{3} .
$$

The invariant RR seems to be first encountered in a paper by Favaron, Mah'eo. The reciprocal Randic index is defined as follows:

$$
\operatorname{RR}(G)=\sum_{r s \in E(G)} \sqrt{d_{r} d_{s}} .
$$

In the same manner, the reduced second Zagreb index (equation (13)), is related to the ordinary second Zagreb index (equation (12)). The reduced reciprocal Randic index might be viewed as the reduced analogue of the reciprocal Randic index (equation (15)):

$$
\operatorname{RRR}(G)=\sum_{r s \in E(G)} \sqrt{\left(d_{r}-1\right)\left(d_{s}-1\right)} .
$$

Vukicevic et al. introduce the symmetric division deg index in $2010[19,20]$ as 


$$
\operatorname{SDD}(G)=\sum_{r s \in E(G)} \frac{d_{r}^{2}+d_{s}^{2}}{d_{r} d_{s}} .
$$

Vukicevic and Gašperov in [20] initiated the study on the inverse sum indeg index of a network. The inverse sum indeg index is defined as follows [21]:

$$
\operatorname{SDD}(G)=\sum_{r s \in E(G)} \frac{d_{r} d_{s}}{d_{r}+d_{s}} .
$$

Another index which belongs to the 4th class of $\mathrm{ABC}$ index was invented by Ghorbani and Hosseinzadeh in 2010 [22] as

$$
\operatorname{ABC}(G)=\sum_{r s \in E(G)} \sqrt{\frac{S_{r}+S_{s}-2}{S_{r} S_{s}}} .
$$

The fifth class of geometric-arithmetic index, denoted by $\mathrm{GA}_{5}$, was presented by Graovac et al. in 2011 [23] as

$$
\mathrm{GA}(G)=\sum_{r s \in E(G)} \frac{2 \sqrt{S_{r} S_{s}}}{S_{r}+S_{s}} .
$$

\section{Main Results of Boron $B_{12}$ Graph}

In this paper, we deal with the topological properties of boron $B_{12}$. Boron $B_{12}$ is a two-dimensional icosahedral network. A recent study of high-pressure solid boron affirmed that the icosahedral $\mathrm{B}_{12}$-containing structures are quite universal [8]. On the other hand, previous experimental and theoretical studies of freestanding boron clusters have shown that the $\mathrm{B}_{12}$ structure is unstable in the gas phase $[9,10]$.

The molecular graph of Boron $B_{12}$ is shown in Figure 1 . There are $11 m n+11 m+11 n+9$ vertices and $24 m n+22 m+$ $22 n+18$ edges.

Theorem 1. Let $G$ be the boron $B_{12}$ network with $m>1$ and $n>1$, then

$$
\begin{aligned}
M_{\alpha}= & 2(2 m+2 n) 2^{\alpha}+(3 m n+m+5) 3^{\alpha} \\
& +(3 m n+2 m+3 n+4) 3^{\alpha}+(m+2 n+1) 4^{\alpha} \\
& +(9 m n+7 m+6 n+5) 4^{\alpha}+(9 m n+7 m+7 n+3) 5^{\alpha} .
\end{aligned}
$$

Proof. In $G$, there are total $11 m n+11 m+11 n+9$ vertices. There are $2 m+2 n$ vertices of degree $2,2 m n+m+n+3$ vertices of degree $3,3 m n+3 m+3 n+3$ vertices of degree 4 , and $36 m n+5 m+5 n+3$ vertices of degree 5 . Since $M_{\alpha}$ is expressed in equation (2),

$$
\begin{aligned}
M_{\alpha}(G)= & \sum_{r \in V(G)}\left(d_{r}\right)^{\alpha} \\
M_{\alpha}= & 2(2 m+2 n) 2^{\alpha}+(3 m n+m+5) 3^{\alpha} \\
& +(3 m n+2 m+3 n+4) 3^{\alpha} \\
& +(m+2 n+1) 4^{\alpha}+(9 m n+7 m+6 n+5) 4^{\alpha} \\
& +(9 m n+7 m+7 n+3) 5^{\alpha} .
\end{aligned}
$$

Proof. Let $G$ be the network of boron $\mathrm{B}_{12}$. The edge set $E(G)$ is distributed in seven categories which depend on the degrees of the end vertices. The first disjoint edge set $E_{1}(G)$

In the following six theorems, we considered the Randic index, sum connectivity index, $\mathrm{ABC}$ index, GA index, harmonic index, reduced Randic index, forgotten index, symmetric division deg index, inverse sum indeg index, $\mathrm{ABC}_{4}$ index, and $\mathrm{GA}_{5}$ index. This is the edge partition of boron $\mathrm{B}_{12}$ on the basis of starting and ending vertices of each edge, and we proceed this edge division to compute the topological indices.

Theorem 2. Let $G$ be the boron $B_{12}$ network with $m>1$ and $n>1$, then

(1) $R_{\alpha}(G)=(2 m+2 n) 8^{\alpha}+(2 m+2 n) 10^{\alpha}+(3 m n+m+$ 5) $12^{\alpha}+(m+2 n+1) 16^{\alpha}+(3 m n+2 m+3 n+4)$ $15^{\alpha}+(9 m n+7 m) 20^{\alpha}+(9 m n+7 m+7 n+3) 25^{\alpha}+$ $(6 n+5) 20^{\alpha}$

(2) $\chi_{\alpha}(G)=(2 m+2 n) 6^{\alpha}+(2 m+2 n) 7^{\alpha}+(3 m n+m+$ $5) 7^{\alpha}+(m+2 n+1) 8^{\alpha}+(3 m n+2 m+3 n+4) 8^{\alpha}+$ $(9 m n+7 m+6 n+5) 9^{\alpha}+(9 m n+7 m+7 n+3) 10^{\alpha}$

(3) $A B C(G)=(2 m+2 n) \sqrt{2}+(1 / 6)(3 m n+m+\quad 5)$ $\sqrt{15}+(1 / 5)(3 m n+2 m+3 n+4) \sqrt{10}+\quad(1 / 4)(m+$ $2 n+1) \sqrt{6}+(1 / 10)(9 m n+7 m) \sqrt{35}+(1 / 10)(6 n+$ 5) $\sqrt{35}+(2 / 5)(9 m n+7 m+7 n+3) \sqrt{2}$

(4) $G A(G)=(2 / 3)(2 m+2 n) \sqrt{2}+(2 / 7)(2 m+2 n) \sqrt{10}+$ $(4 / 7)(3 m n+m+5) \sqrt{3}+(1 / 4)(3 m n+2 m+3 n+$ 4) $\sqrt{15}+(4 / 9)(9 m n+7 m+6 n+5) \sqrt{5}+(9 m n+$ $8 m+9 n+4)$

Proof. The network of boron $\mathrm{B}_{12}$ has $24 m n+22 m+22 n+$ 18 number of edges. There are seven disjoint edge sets of i.e., $\quad E(G)=E_{1}(G) \cup E_{2}(G) \cup E_{3}(G) \cup \quad E_{4}(G) \cup E_{5}(G) \cup$ $E_{6}(G) \cup E_{7}(G)$. The edge partition $E_{1}(G)$ holds $2 m+2 n$ edges $r s$, where $d_{r}=2$ and $d_{s}=4$, the edge partition $E_{2}(G)$ holds $2 m+2 n$ edges $r s$, where $d_{r}=2$ and $d_{s}=5$, the edge partition $E_{3}(G)$ holds $3 m n+m+5$ edges $r s$, where $d_{r}=3$ and $d_{s}=4$, the edge partition $E_{4}(G)$ holds $3 m n+2 m+3 n+$ 4 edges $r$, where $d_{r}=3$ and $d_{s}=5$, the edge partition $E_{5}(G)$ holds $m+2 n+1$ edges $r s$, where $d_{r}=d_{s}=4$, the edge partition $E_{6}(G)$ holds $9 m n+7 m+6 n+5$ edges $r s$, where $d_{r}=4$ and $d_{s}=5$, and the edge partition $E_{7}(G)$ holds $9 m n+$ $7 m+7 n+3$ edges $r s$, where $d_{r}=d_{s}=5$. From formulas (1) and (3)-(5), we get the desired results.

Theorem 3. Let $G$ be the boron $B_{12}$ network with $m>1$ and $n>1$, then

(1) $H M(G)=1678 m+1676 n+1968 m n+1270$

(2) $P M_{1}(G)=6^{2 m+2 n} \times 7^{2 m+2 n} \times 7^{3 m n+m+5} \times 8^{3 m n+2 m+3 n+4}$ $\times 8^{m+2 n+1} \times 9^{9 m n+7 m+6 n+5} \times 10^{9 m n+7 m+7 n+3}$

(3) $P M_{2}(G)=8^{2 m+2 n} \times 10^{2 m+2 n} \times 12^{3 m n+m+5} \times$ $15^{3 m n+2 m+3 n+4} \times 16^{m+2 n+1} \times 20^{9 m n+7 m+6 n+5} \times 25^{9 m n+7 m+7 n+3}$ edge set $E(G)$ depending on the degrees of the end vertices, 


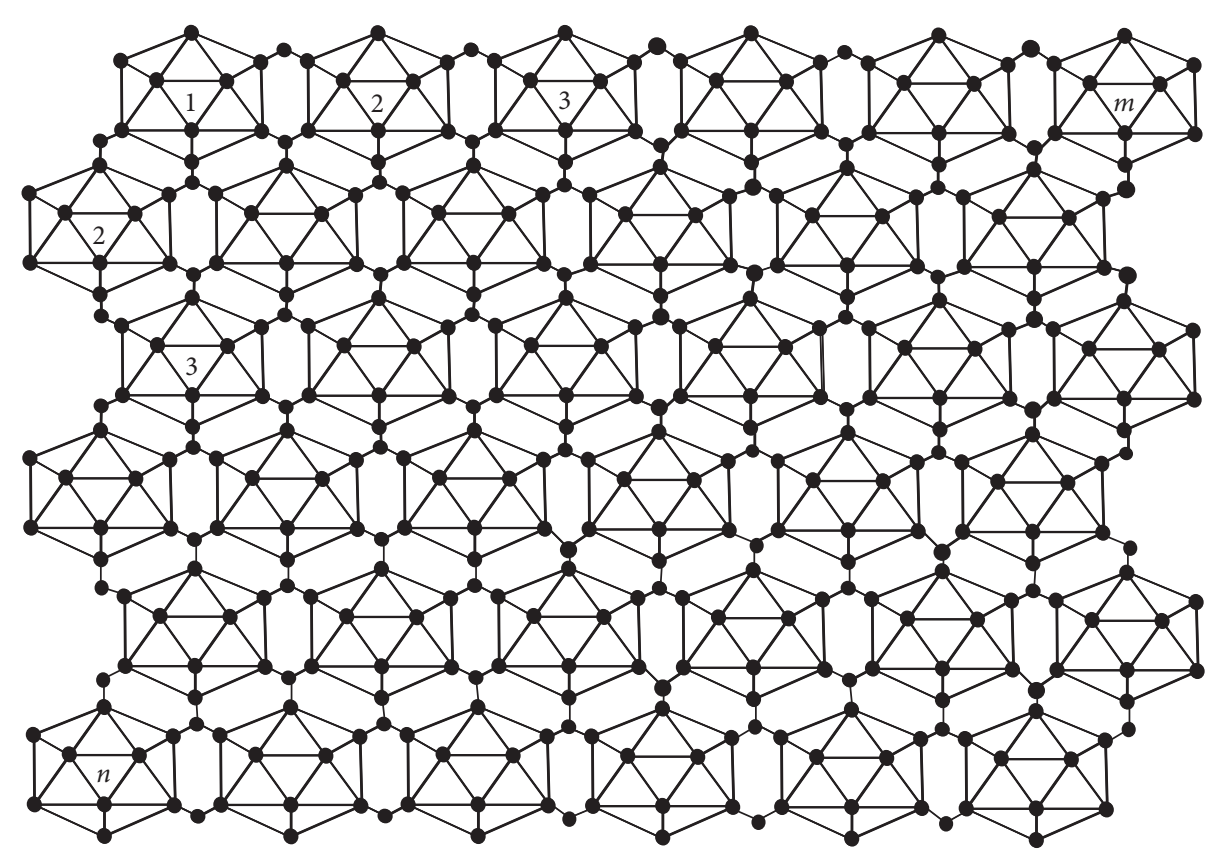

Figure 1: $(n, m)$ unit of boron.

holds $2 m+2 n$ edges $r s$, where $d_{r}=2$ and $d_{s}=4$, the second disjoint edge set $E_{2}(G)$ holds $2 m+2 n$ edges $r s$, where $d_{r}=2$ and $d_{s}=5$, the third disjoint edge set $E_{3}(G)$ holds $3 m n+$ $m+5$ edges $r s$, where $d_{r}=3$ and $d_{s}=4$, the forth disjoint edge set $E_{4}(G)$ holds $3 m n+2 m+3 n+4$ edges $r s$, where $d_{r}=$ 3 and $d_{s}=5$, the fifth disjoint edge set $E_{5}(G)$ holds $m+$ $2 n+1$ edges $r s$, where $d_{r}=d_{s}=4$, the sixth disjoint edge set $E_{6}(G)$ holds $9 m n+7 m+6 n+5$ edges $r s$, where $d_{r}=4$ and $d_{s}=5$, and the seventh disjoint edge set $E_{7}(G)$ holds $9 m n+$ $7 m+7 n+3$ edges $r s$, where $d_{r}=d_{s}=5$. Now, $\left|E_{1}(G)\right|=e_{2,4},\left|E_{2}(G)\right|=e_{2,5},\left|E_{3}(G)\right|=e_{3,4},\left|E_{4}(G)\right|=e_{3,5}$, $\left|E_{5}(G)\right|=e_{4,4},\left|E_{6}(G)\right|=e_{4,5}$, and $\left|E_{7}(G)\right|=e_{5,5}$. We obtained results by using formulas (6), (9), and (10).

Theorem 4. Let $G$ be the boron $B_{12}$ network with $m>1$ and $n>1$, then

(1) $M_{1}(G, p)=(2 m+2 n) p^{6}+(2 m+2 n) p^{7}+(3 m n+m$ $+5) p^{7}+(m+2 n+1) p^{8}+\left((3 m n+2 m) p^{8}+(3 n\right.$ $+4)) p^{8}+(9 m n+7 m+6 n+5) p^{9}+(9 m n+7 m+$ $7 n+3) p^{10}$

(2) $M_{1}(G, p)=(2 m+2 n) p^{8}+(2 m+2 n) p^{10}+(3 m n+$ $m+5) p^{12}+\left((3 m n+2 m) p^{15}+(3 n+4)\right) p^{15}+(m+$ $2 n+1) p^{16}+(9 m n+7 m+6 n+5) p^{20}+(9 m n+$ $7 m+7 n+3) p^{25}$

Proof. Let $G$ be the network of boron $\mathrm{B}_{12}$. The edge set $E(G)$ is distributed in seven categories which depend on the degree of end vertices of each edge. The disjoint set is represented by $e_{r, s}$. The first disjoint set is $e_{2,4}$, the second disjoint set is $e_{2,5}$, the third disjoint set is $e_{3,4}$, the fourth disjoint set is $e_{3,5}$, the fifth disjoint set is $e_{4,4}$, the sixth disjoint set is $e_{4,5}$, and the seventh disjoint set is $e_{5,5}$. By using formulas (11) and (12), we obtained the required results.
Theorem 5. Let $G$ be the boron $B_{12}$ network with $m>1$ and $n>1$, then

(1) $R M_{2}(G)=241 m+240 n+294 m n+179$

(2) $A Z I(G)=(12576690219 / 21952000) m n+(4004353$ $2353 / 84672000) m+(2230300883 / 4741632) n+(42$ $526959271 / 118540800)$

Proof. Let $G$ be the network of boron $\mathrm{B}_{12}$. The edge set $E(G)$ is distributed in seven categories which depend on the degree of end vertices of each edge. The disjoint set is represented by $e_{r, s}$. The first disjoint set is $e_{2,4}$, the second disjoint set is $e_{2,5}$, the third disjoint set is $e_{3,4}$, the fourth disjoint set is $e_{3,5}$, the fifth disjoint set is $e_{4,4}$, the sixth disjoint set is $e_{4,5}$, and the seventh disjoint set is $e_{5,5}$. We get the results by using formulas (13) and (14).

Theorem 6. Let $G$ be the boron $B_{12}$ network with $m>1$ and $n>1$, then

(1) $H(G)=(6589 / 1260) m+(731 / 140) n+(757 / 140)$ $m n+(5531 / 1260)$

(2) $R R(G)=2(2 m+2 n) \sqrt{2}+(2 m+2 n) \sqrt{10}+2(3 m n+$ $m+5) \sqrt{3}+((3 m n+2 m) \sqrt{15}+(3 n+4)) \sqrt{15}+$ $2(9 m n+7 m+6 n+5) \sqrt{5}+45 m n+39 m+43 n+19$

(3) $\operatorname{RRR}(G)=(2 m+2 n) \sqrt{3}+2(3 m n+2 m \sqrt{2}+2(3 n$ +4) $\sqrt{2}+2(3 m n+m+5) \sqrt{6}+2(9 m n+7 m+6 n+$ 5) $\sqrt{3}+36 m n+35 m+38 n+15$

Proof. Let $G$ be the network of boron $\mathrm{B}_{12}$. The edge set $E(G)$ is distributed in seven categories which depend on the degree of end vertices of each edge. The disjoint set is represented by $e_{r, s}$. The first disjoint set is $e_{2,4}$, the second disjoint set is $e_{2,5}$, the third disjoint set is $e_{3,4}$, the fourth 
disjoint set is $e_{3,5}$, the fifth disjoint set is $e_{4,4}$, the sixth disjoint set is $e_{4,5}$, and the seventh disjoint set is $e_{5,5}$. By using formulas (7), (15), and (16), we get the desired results.

Theorem 7. Let $G$ be the boron $B_{12}$ network with $m>1$ and $n>1$, then

(1) $F(G)=860 m+860 n+996 m n+648$

(2) $\operatorname{SDD}(G)=(1433 / 30) m+(479 / 10) n+(99 / 2) m n+$ $(566 / 15)$

(3) $I S I(G)=(11603 / 252) m+(2575 / 25562) n+(2983 /$ $56) m n+(2311 / 63)$

Proof. Let $G$ be the network of boron $\mathrm{B}_{12}$. The edge set $E(G)$ is distributed in seven categories which depend on the degree of end vertices of each edge. The disjoint set is represented by $e_{r, s}$. The first disjoint set is $e_{2,4}$, the second disjoint set is $e_{2,5}$, the third disjoint set is $e_{3,4}$, the fourth disjoint set is $e_{3,5}$, the fifth disjoint set is $e_{4,4}$, the sixth disjoint set is $e_{4,5}$, and the seventh disjoint set is $e_{5,5}$. We obtained the required results by using formulas (8), (17), and (18).

In the following two theorems, we considered the fourth atom-bond connectivity index and the fifth geometricarithmetic index. This is the edge partition of boron $B_{12}$ on the basis of the degree sum of neighbors of end vertices of each edge. We proceed this edge division to compute $\mathrm{ABC}_{4}(G)$ and $\mathrm{GA}_{5}(G)$

Theorem 8. Let $G$ be the boron $B_{12}$ network with $m>1$ and $n>1$, then

$$
\begin{aligned}
\mathrm{ABC}_{4}(G)= & (1 / 2) \sqrt{2}+(4 / 221) \sqrt{1547}+(4 / 273) \sqrt{546}-(2 / 3)+(2 / 22) \sqrt{22} \\
& +(1 / 22) \sqrt{42}+(1 / 10)(m-1) \sqrt{10}+(1 / 114)(m+2 n+1) \sqrt{1330}+(1 / 68)(m-1) \sqrt{442} \\
& +(1 / 391)(m+3) \sqrt{14858}+(1 / 374)(m+1) \sqrt{13838}+(1 / 34)(m-1) \sqrt{119}+(2 / 119)(m+1) \sqrt{357} \\
& +(1 / 92)(m+2 n+1) \sqrt{851}+(1 / 12)(m+2 n+1) \sqrt{15}+(1 / 68)(m+3) \sqrt{527}+(2 / 115)(n-1) \sqrt{345} \\
& +(4 / 285)(2 n-2) \sqrt{570}+(1 / 238)(m+1) \sqrt{6902}+(1 / 266)(m+3) \sqrt{8246}+(1 / 154)(m+3) \sqrt{2618} \\
& +(1 / 10)(m-1) \sqrt{14}+(1 / 8)(m+2 n+1) \sqrt{11}+(1 / 68)(m-1) \sqrt{782}+(3 / 190)(m+2 n+1) \sqrt{570}+(1 / 6) m n \sqrt{30} \\
& +(1 / 35) m n \sqrt{1190}+(1 / 6) m n \sqrt{42}+(1 / 42)(3 m n-2 n-1) \sqrt{46}+(1 / 92)(m+4 n-3) \sqrt{690}+(2 / 3) n \sqrt{11}+(1 / 6)(m+3) \sqrt{3} \\
& +(1 / 506)(m+1) \sqrt{21758}+(1 / 84)(6 m n-m-2 n-1) \sqrt{602}+(1 / 23)(m+2 n+3) \sqrt{46}+(1 / 20)(2 m-2) \sqrt{35} \\
& +(1 / 228)(m+2 n+1) \sqrt{4674}+(2 / 437)(2 n-2) \sqrt{4370}+(1 / 418)(m+3) \sqrt{16302}+(1 / 138)(2 n-2) \sqrt{1794} \\
& +(1 / 126)(6 m n-2 m-2 n) \sqrt{1554}+(2 / 3) n .
\end{aligned}
$$

Proof. The network of boron $\mathrm{B}_{12}$ has $24 m n+22 m+22 n+$ 18 number of edges. There are 41 disjoint degree sum of neighbors of end vertices of each edge, i.e., $e_{8,16}, e_{8,17}, e_{10,19}$, $e_{10,20}, e_{12,18}, \ldots, e_{24,24}$. By using formula (19), we get the desired result.
Theorem 9. Let $G$ be the boron $B_{12}$ network with $m>1$ and $n>1$, then

$$
\begin{aligned}
\mathrm{GA}_{5}(G)= & (1 / 20)(m+3) \sqrt{391}+(1 / 19)(m+1) \sqrt{357}+(1 / 39)(m+1) \sqrt{374}+(1 / 19)(n-1) \sqrt{345} \\
& +(1 / 17)(2 n-2) \sqrt{285}+(2 / 29)(m+2 n+1) \sqrt{190}+(2 / 31)(m+1) \sqrt{238}+(2 / 33)(m+3) \sqrt{266} \\
& +(2 / 41)(m+3) \sqrt{418}+(1 / 21)(2 n-2) \sqrt{437}+(1 / 22)(m+2 n+3) \sqrt{483}+(2 / 45)(m+1) \sqrt{506} \\
& +(2 / 15) \sqrt{273}+(2 / 13)(6 m n-2 m-2 n) \sqrt{42}+(6 / 37)(m+2 n+1) \sqrt{38}+(6 / 19)(m-1) \sqrt{10}+(4 / 41)(m-1) \sqrt{102} \\
& +(8 / 37)(m+2 n+1) \sqrt{21}+(4 / 37)(m-1) \sqrt{85}+(8 / 39)(m+2 n+1) \sqrt{23}+(8 / 33)(m+3) \sqrt{17}+(12 / 17)(2 n-2) \sqrt{2} \\
& +(2 / 3)(m-1) \sqrt{2}+(1 / 9)(m+3) \sqrt{77}+(2 / 3)(m+2 n+1) \sqrt{2}+(4 / 25)(m-1) \sqrt{34}+(12 / 7) m n \sqrt{3}+(1 / 2) m n \sqrt{35} \\
& +(6 / 5) m n \sqrt{6}+(6 / 41)(2 n-2) \sqrt{46}+(2 / 11)(2 m-2) \sqrt{30}+(4 / 43)(m+2 n+1) \sqrt{114}+(4 / 15)(6 m n-m-2 n-1) \sqrt{14} \\
& +(4 / 23)(m+3) \sqrt{33}+(4 / 47)(m+4 n-3) \sqrt{138}+(8 / 15) \sqrt{14}+(8 / 19) \sqrt{22}-n+3 m n .
\end{aligned}
$$


Proof. The network of boron $\mathrm{B}_{12}$ has $24 m n+22 m+22 n+$ 18 number of edges. There are 41 disjoint degree sum of neighbors of end vertices of each edge, i.e., $e_{8,16}, e_{8,17}, e_{10,19}$, $e_{10,20}, e_{12,18}, \ldots, e_{24,24}$. By using formula (20), we get the desired result.

\section{Conclusion}

We have computed the following topological indices such as Zagreb index, the Randic index, sum connectivity index, ABC index, GA index, hyper-Zagreb index, multiple Zagreb indices, Zagreb polynomials, reduced second Zagreb index, augmented Zagreb index, harmonic index, reduced Randic index, reduced reciprocal Randic index, forgotten index, $\mathrm{ABC}_{4}$ index, and $\mathrm{GA}_{5}$ index for boron $\mathrm{B}_{12}$ structure.

In cheminformatics, Randic index is used to study the organic compounds. It is correlated with physicochemical properties of alkane such as boiling points, surface area, and enthalpy of formation. ABC index is based on some physicochemical properties like the stability of cyclo-alkane as well as strain energy. GA index has more predictive power than Randic and ABC index. Multiple Zagreb indices and Zagreb polynomials are applied to predict the bioactivity of graphs. The forgotten index is correlated with some chemical properties relating to energies of molecular graphs. AZI index is a valuable predictive index which is used in the study of the heat of formation. RRR index is used to study the normal boiling points of the graphs.

We have discussed the graph theoretically not experimentally. The results obtained in this paper provide a significant contribution to graph theory and correlate the chemical structure of boron $B_{12}$ with the large amount of information about physicochemical properties.

\section{Data Availability}

No data were used to support this study.

\section{Conflicts of Interest}

The authors declare that they have no conflicts of interest.

\section{Acknowledgments}

The authors wish to acknowledge the financial support from the National Natural Science Foundation of Beijing (2182067) and the Fundamental Research Funds of the Central Universities (2018ZD09).

\section{References}

[1] H. M. A. Siddiqui, "Computation of Zagreb indices and Zagreb polynomials of Sierpinski graphs," Hacettepe Journal of Mathematics and Statistics, vol. 49, no. 2, pp. 754-765, 2020.

[2] H. M. A. Siddiqui, S. Baby, M. K. Shafiq, and M. Faisal Nadeem, "Bounds of some degree based indices of lexicographic product of some connected graphs," Polycyclic Aromatic Compounds, 2020.

[3] J.-B. Liu, B. Ali, M. Aslam Malik, H. M. A. Siddiqui, and M. Imran, "Reformulated Zagreb indices of some derived graphs," Mathematics, vol. 7, p. 283, 2019.
[4] B. L. Jia, M. Imran, S. Baby, H. M. A. Siddiqui, and M. K. Shafiq, "Graph indices for Cartesian product of F-sum of connected graphs," Combinatorial Chemistry \& High Throughput Screening, 2021.

[5] J. Xu, J.-B. Liu, A. Bilal et al., "Distance degree index of some derived graphs," Mathematics, vol. 7, no. 3, p. 283, 2019.

[6] M. Ghorbani and N. Azimi, "Note on multiple Zagreb indices," Iranian Journal of Mathematical Chemistry, vol. 3, no. 2, pp. 137-143, 2012.

[7] I. Gutman, "Degree-based topological indices," Croatica Chemica Acta, vol. 86, no. 4, pp. 351-361, 2013.

[8] A. R. Oganov, J. Chen, C. Gatti et al., "Ionic high-pressure form of elemental boron," Nature, vol. 457, no. 7231, pp. 863-867, 2009.

[9] I. Boustani, "Systematic ab initio investigation of bare boron clusters: mDetermination of the geometry and electronic structures of $\mathrm{B} n(n=2-14)$," Physical Review B, vol. 55, no. 24, p. $16426,1997$.

[10] H. Li, N. Shao, B. Shang, L.-F. Yuan, J. Yang, and X. C. Zeng, "Icosahedral B12-containing core-shell structures of B80," Chemical Communications, vol. 46, no. 22, pp. 3878-3880, 2010.

[11] L. W. Beineke and R. J. Wilson, Applications of Graph Theory, Academic Press, London, UK, 1979.

[12] B. Horoldagva, L. Buyantogtokh, K. C. Das, and S. G. Lee, "On general reduced second Zagreb index of graphs," Hacettepe Journal of Mathematics and Statistics, vol. 48, no. 4, pp. 1040-1056, 2019.

[13] K. C. Das, I. Gutman, and B. Furtula, "On atom-bond connectivity index," Chemical Physics Letters, vol. 511, no. 4-6, pp. 452-454, 2011.

[14] K. C. Das, "On geometric-arithmetic index of graphs," MATCH Communications in Mathematical and in Computer Chemistry, vol. 64, no. 3, pp. 619-630, 2010.

[15] D. Vukievi and B. Furtula, "Topological index based on the ratios of geometrical and arithmetical means of end-vertex degrees of edges," Journal of Mathematical Chemistry, vol. 46, no. 4, pp. 1369-1376, 2009.

[16] S. Fajtlowicz, "Harmonic index," Congressus Numerantium, vol. 60, pp. 187-197, 1987.

[17] Z. Shao, M. Siddiqui, and M. Muhammad, "Computing Zagreb indices and Zagreb polynomials for symmetrical nanotubes," Symmetry, vol. 10, no. 7, p. 244, 2018.

[18] A. Ali, Z. Raza, and A. A. Bhatti, "On the augmented Zagreb index," 2014, http://arxiv.org/abs/1402.3078.

[19] D. Vukievi, "Bond additive modeling 2. mathematical properties of max-min Rodeg index," Croatica Chemica Acta, vol. 83, no. 3, pp. 261-273, 2010.

[20] D. Vukievi and M. Gašperov, "Bond additive modeling 1. adriatic indices," Croatica Chemica Acta, vol. 83, no. 3, pp. 243-260, 2010.

[21] J. Sedlar, D. Stevanović, and A. Vasilyev, "On the inverse sum indeg index," Discrete Applied Mathematics, vol. 184, pp. 202-212, 2015.

[22] M. Ghorbani and M. A. Hosseinzadeh, "Computing ABC4 index of nanostar dendrimers," Optoelectronics and Advanced Materials-Rapid Communications, vol. 4, no. 9, pp. 1419-1422, 2010.

[23] A. Graovac, M. Ghorbani, and M. A. Hosseinzadeh, "Computing fifth geometric-arithmetic index for nanostar dendrimers," Journal of Mathematical Nanoscience, vol. 1, no. 1-2, pp. 33-42, 2011. 